\title{
LA GESTIONE DEL CICLO DELLE ACQUE TRA STORIA, SCIENZA E LETTERATURA
}

\author{
STEFANO POLESELLO $(*)$
}

Nota presentata dal m.e. C. Fuganti

(Adunanza del 1 febbraio 2018)

SUNTO. - Questa conversazione vuole presentare un excursus sul tema del rapporto tra acqua e uomo e della gestione dell'acqua tra storia, letteratura e scienza, dallo sviluppo delle prime civiltà alle più recenti conseguenze delle attività umane sugli ecosistemi acquatici, mostrando le soluzioni più recenti proposte per la gestione moderna del ciclo delle acque anche alla luce dei cambiamenti globali in corso.

$$
* * *
$$

ABSTRACT. - This lecture aims to present an excursus on the theme of the relationship between water and man and the management of water between history, literature and science, from the development of the first civilizations to the most recent consequences of human activities on aquatic ecosystems, showing the most recent solutions proposed for modern management of the water cycle also in light of the global change underway.

(*) Istituto di Ricerca sulle Acque del CNR, sede di Brugherio (MB), Italy. E-mail: polesello@irsa.cnr.it 


\title{
1. L'ACQUA: DALLA COSMOGONIA DELL'UNIVERSO ALL'OSSERVAZIONE NATURALISTICA
}

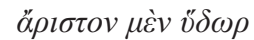 \\ Ottima è l'acqua, l'oro come fuoco acceso \\ nella notte sfolgora sull'esaltante ricchezza
}

\section{Pindaro - Olimpiche}

È nozione comune fin dall'infanzia che la molecola dell'acqua sia indispensabile alla vita, ma non per tutti è ovvio che questo è dovuto alle sue intrinseche caratteristiche chimico-fisiche, cioè a come sono connessi gli atomi nella molecola e come vi è distribuita la densità elettronica. L'acqua è indispensabile alla vita perché è il miglior solvente e quindi il miglior mezzo di trasporto delle sostanze chimiche (sali, zuccheri ed aminoacidi, per esempio) indispensabili ai processi biologici catabolici e metabolici. Questo è possibile perché l'acqua ha un'elevata costante dielettrica (cioè la capacità di separare le cariche elettriche, proprietà indispensabile per allontanare gli ioni dal reticolo cristallino e quindi sciogliere le sostanze ioniche cioè i sali) ed ha la possibilità di formare forti legami a idrogeno con gruppi idrossilici $(\mathrm{OH})$ presenti ad esempio negli zuccheri, solvatando così le molecole o gli ioni solubilizzati.

Che l'acqua fosse indispensabile alla vita è sempre stato evidente all'uomo che ha costruito sui fiumi le più grandi e antiche civiltà. La stessa topografia del giardino dell'Eden viene descritta in Genesi 2,1014 in base alla sua idrografia [1]:

\footnotetext{
${ }^{10}$ Un fiume usciva da Eden per irrigare il giardino, poi di li si divideva e formava quattro corsi. ${ }^{11}$ Il primo fiume si chiama Pison: esso scorre intorno a tutto il paese di Avila, dove c'è l'oro ${ }^{12}$ e l'oro di quella terra è fine; qui c'è anche la resina odorosa e la pietra d'ònice. ${ }^{13} \mathrm{Il}$ secondo fiume si chiama Ghicon: esso scorre intorno a tutto il paese d'Etiopia. ${ }^{14}$ Il terzo fiume si chiama Tigri: esso scorre ad oriente di Assur. Il quarto fiume è l'Eufrate.
}

La prima Genesi della tradizione ebraica è simile alle cosmogonie mesopotamiche, che cominciano con l'emersione della terra dalle acque primordiali del caos e, metaforicamente, narrano come il suolo asciutto sorgesse dagli straripamenti annuali di Tigri ed Eufrate. Da un antico racconto della creazione di Ebla si dice addirittura che fu allora che gli dei furono formati dal seno delle acque [2]. La narrazione della creazio- 
ne nei primi versetti della Genesi (Gn 1, 1-10) non narra in modo esplicito della creazione dell'acqua, che anzi è la materia primordiale su cui aleggia lo spirito di Dio (Fig. 1):

${ }^{1}$ In principio Dio creò il cielo e la terra. ${ }^{2}$ Ora la terra era informe e deserta e le tenebre ricoprivano l'abisso e lo spirito di Dio aleggiava sulle acque. [...] ${ }^{6}$ Dio disse: "Sia il firmamento in mezzo alle acque per separare le acque dalle acque". 'Dio fece il firmamento e separò le acque, che sono sotto il firmamento, dalle acque, che son sopra il firmamento. E cosi avvenne. ${ }^{8}$ Dio chiamò il firmamento cielo. E fu sera e fu mattina: secondo giorno. 'Dio disse: "Le acque che sono sotto il cielo, si raccolgano in un solo luogo e appaia l'asciutto". E cosi avvenne. ${ }^{10}$ Dio chiamò l'asciutto terra e la massa delle acque mare.

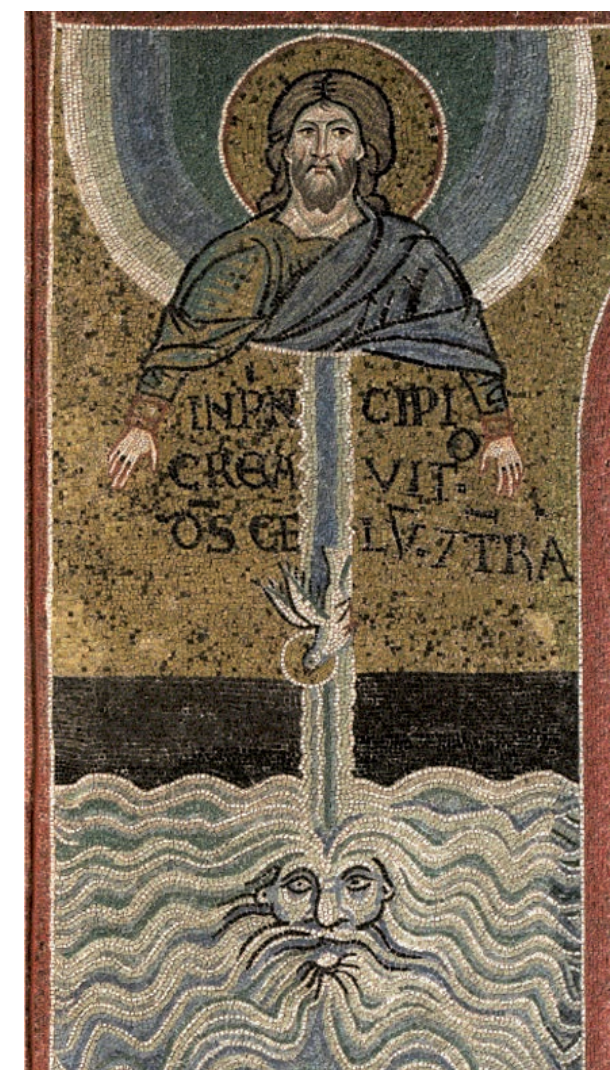

Fig. 1. Lo spirito di Dio aleggia sulle acque. Mosaico, Cattedrale di Monreale. 
Per la Bibbia l'acqua ha un valore ambivalente: è il dono che come la parola di Dio scende dal cielo e feconda la terra (Is 55, 10-11), e allo stesso tempo può rappresentare l'abisso primordiale del caos che può inghiottire l'uomo. Il mare è il luogo stesso del Male da cui la Bestia dell'Apocalisse (Ap 13,1), il luogo dove viene gettato e ingoiato il profeta Giona per tre giorni (Gio 1,15). L'acqua può distruggere l'umanità come nel Diluvio o far nascere a vita nuova mediante il battesimo.

Questo ha ovviamente conferito all'acqua un valore altamente simbolico che ha condizionato il pensiero umano sull'acqua fino alla nascita del pensiero moderno. Un pensatore come Dante, ponendosi un problema scientifico sull'acqua nella Quaestio de Aqua et Terra [3], non riesce ad astrarsi da una visione cosmologica anche quando cerca di interpretare osservazioni ed evidenze dei "sensi". La questione dibattuta in questo breve libro riguardava il luogo e la figura o (meglio) la forma dei due elementi dell'acqua e della terra:

se l'acqua nella sua sfera, cioè nella sua superficie naturale, fosse in qualche parte più alta della terra che emerge dalle acque e che noi comunemente chiamiamo la «quarta (parte) abitabile»

Dante, nella confutazione delle varie argomentazioni degli avversari, utilizza però nella parte finale anche le evidenze sensibili, mediate dall'autorità del Filosofo per eccellenza.

Infatti il volgo e le persone che ignorano i trattati dei fisici credono che l'acqua salga, proprio sotto forma di acqua, fino alle cime dei monti e al luogo delle sorgenti, ma ciò è assai puerile, poiché quivi le acque si formano per l'evaporazione della materia, come risulta dalla Meteorologia del Filosofo.

Quindi in realtà, grazie alla divulgazione delle opere di Aristotele, nella cosmologia medievale, basata sulla Genesi, si innesta una prima intuizione del ciclo dell'acqua come evaporazione, condensazione, pioggia che forma i corsi d'acqua.

Bisogna aspettare Leonardo per avere un primo "ricercatore" dedicato ai temi dell'acqua, con la sua capacità di osservare, descrivere e disegnare tutti i fenomeni naturali, ma anche le sue limitazioni nel dare ordine sistematico, in un modo che noi chiameremmo scientifico, alle sue osservazioni e intuizioni. Leonardo già aveva intuito che il sistema acqua doveva essere studiato come un tutt'uno, che ciascuna parte 
del ciclo delle acque era collegata all'altra, che ogni azione anche limitata ad una parte aveva effetto sulle altre. Leonardo progettò un trattato di cui scrisse l'indice e i contenuti principali, ma che, come per gli altri trattati, non venne mai concluso e pubblicato.

\section{L’ACQUA COME REFLUO E LA SUA GESTIONE}

L'égout, c'est la conscience de la ville. Tout y converge et s'y confronte.

\section{Hugo, Les Misérables}

\subsection{Il collettamento dei reflui urbani}

Il nostro immaginario dell'antichità è legato alle grandi opere idrauliche di adduzione dell'acqua delle grandi civiltà antiche che in molti casi caratterizzano ancora il paesaggio urbano e rurale del Mediterraneo. Il nostro pensiero corre immediatamente alle arcate degli acquedotti romani, o alla rete di gallerie scavate nel tufo di Napoli o alla cisterna dalle mille colonne di Istanbul. Queste grandi opere ingegneristiche nascevano dalla necessità di collettare e accumulare l'acqua anche in città che ne erano prive $o$ in previsioni di assedi. La stessa Roma, pur attraversata da un fiume di discreta portata, necessitava di acqua pulita condotta da aree collinari o montuose distanti decine di chilometri.

La pianificazione urbanistica della distribuzione dell'acqua attraverso il sistema acquedottistico nasceva dalla consapevolezza che la qualità dell'acqua superficiale, specie in una zona intensamente urbanizzata, non garantiva la salubrità sufficiente per la potabilità. Quello che colpisce dell'ingegneria idraulica romana è non solo il sistema di adduzione e distribuzione dell'acqua "pulita", ma soprattutto la gestione, che oggi definiremmo integrata, del ciclo dell'acqua dalla sorgente allo scarico controllato delle acque reflue. Fin dall'epoca repubblicana a Roma vennero effettuati ingenti investimenti per la creazione di un sistema di collettamento e smaltimento delle acque luride. Per far ciò era necessario avere a disposizione grandi quantitativi di acqua pulita con una portata alta e costante che solo gli acquedotti potevano fornire. Una dello loro funzioni principali, meno nota ma altrettanto importante, era il lavaggio in continuo delle strade tramite lo stramazzo dei 
castelli di distribuzione [4]. Questi flussi costanti di acqua venivano convogliati verso le cloache passando attraverso le latrine pubbliche, svolgendo una fondamentale opera di sanità pubblica. La più grande $\mathrm{e}$ famosa tra le cloache era la Cloaca Maxima, il cui sbocco nel Tevere è ancora visibile nei pressi del Ponte Rotto, anche se in realtà essa aveva lo scopo di drenare le acque di scolo naturale del Palatino per evitare l'impaludamento della zona dei Fori Imperiali e del Velabro. La forza dell'organizzazione imperiale era la capacità di riprodurre questi modelli di gestione idraulici anche nei centri urbani di tutto il territorio governato da Roma. Che il sistema fognario fosse uno degli aspetti più impressionanti della gestione urbana erano già coscienti i commentatori antichi come Strabone che nella Geografia (V.3.8) elogia i Romani per "aver pensato a pavimentare vie, a incanalare acque, a costruire fogne che potessero evacuare nel Tevere tutti i rifiuti della citta”.

Nonostante le grandi latrine pubbliche a più posti "a sedere" con impianti di lavaggio (Fig. 2), sistemi fognari, una specifica legislazione igienico-sanitaria, fontane e acqua potabile portata con acquedotti, le misure di igiene pubblica erano insufficienti per proteggere la popolazione dai parassiti diffusi dalla contaminazione fecale come dimostrato da studi recenti nei resti di latrine di città romane [5].

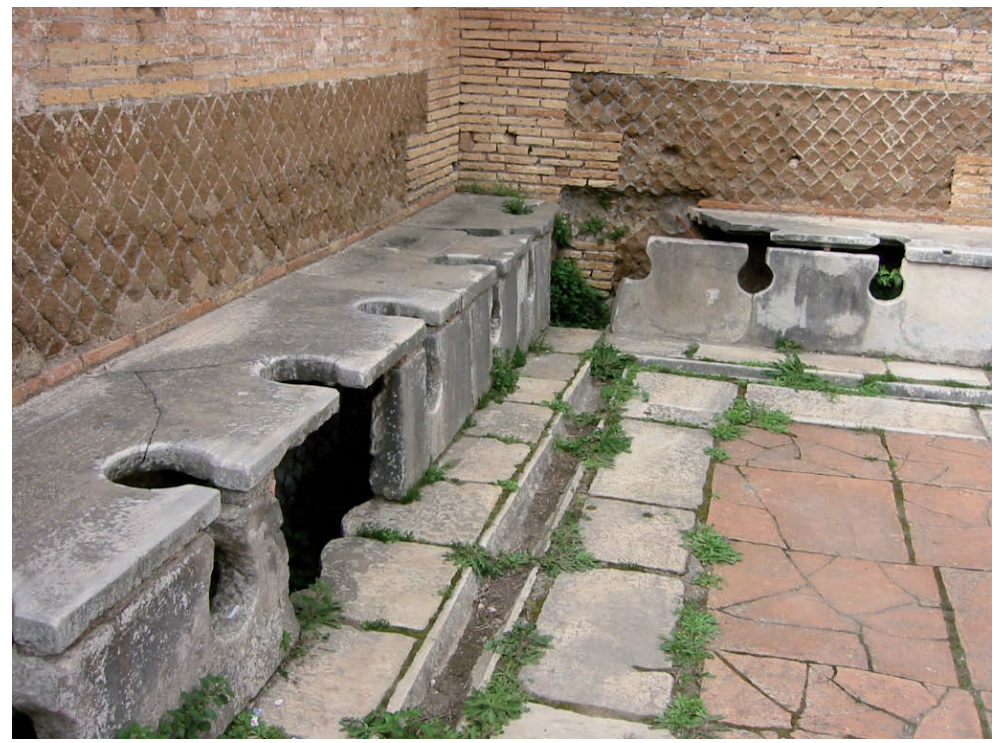

Fig. 2. Latrine di epoca romana negli scavi di Ostia. 
Se la gestione delle deiezioni umane e dei reflui fognari era comunque insufficiente nell'antica Roma a garantire la salubrità pubbli$\mathrm{ca}$, non riusciamo neppure ad immaginare che cosa potessero essere in termini di odore, sporcizia e rischio infettivo le città medievali che avevano lasciato andare in rovina gli antichi impianti di collettamento. Anche nei comuni italiani più avanzati nel Medioevo il collettamento avveniva attraverso canalette che scorrevano a cielo aperto nelle strade. Celebre ed esemplificativo è il passo della novella di Andreuccio da Perugia del Boccaccio [6]:

Era il caldo grande: per la qual cosa Andreuccio, veggendosi solo rimasto, subitamente si spogliò in farsetto e trassesi i panni di gamba e al capo del letto gli si pose; e richiedendo il naturale uso di dovere diporre il superfluo peso del ventre, dove ciò si facesse domandò quel fanciullo, il quale nell'uno de'canti della camera gli mostrò uno uscio e disse: - Andate là entro -. Andreuccio dentro sicuramente passato, gli venne per ventura posto il piè sopra una tavola, la quale dalla contraposta parte sconfitta dal travicello sopra il quale era; per la qual cosa capolevando questa tavola con lui insieme se n'andò quindi giuso: e di tanto l'amò Idio, che niuno male si fece nella caduta, quantunque alquanto cadesse da alto, ma tutto della bruttura, della quale il luogo era pieno, s'imbrattò. Il quale luogo, acciò che meglio intendiate e quello che è detto e ciò che segue, come stesse vi mostrerò. Egli era in un chiassetto stretto, come spesso tra due case veggiamo: sopra due travicelli, tra l'una casa e l'altra posti, alcune tavole eran confitte e il luogo da seder posto, delle quali tavole quella che con lui cadde era l'una.

\subsection{La costruzione dei moderni sistemi fognari}

L'impulso decisivo alla costruzione del sistema fognario nelle grandi città europee furono le epidemie di colera tra il 1830 e la fine del secolo. Nel 1854 per la prima volta il medico inglese John Snow mise in correlazione la diffusione del colera con l'inquinamento dell'acqua di una fontana a SoHo, Londra, causata dalla contiguità tra pozzi neri e pozzi potabili, oltre all'uso di acque potabili prelevate dal fiume Tamigi.

Dal 1852 iniziò la costruzione dei sistemi fognari moderni di Londra e Parigi che ancora oggi sono l'ossatura dei loro impianti fognari. Alcune città italiane (tra le prime Firenze e Milano) iniziarono la costruzione dei nuovi sistemi fognari dopo l'unità d'Italia.

Il nuovo sistema fognario di Parigi diventa addirittura protagonista di un intero capitolo de "I Miserabili" di Victor Hugo [7]: 
Parigi ha sotto di sé un'altra Parigi; una Parigi di fogne, con le vie, $i$ crocicchi, le piazze, i vicoli, le arterie, la sua circolazione, fatta di fango al quale manca, però, la forma umana.

Oggi la fogna è linda, fredda, rigida, corretta, e realizza quasi l'ideale di ciò che s'intende in Inghilterra colla parola «respectable».

Un vero libello ambientale, riflette sia le preoccupazioni dello scrittore che del politico: partendo da una descrizione dantesca di questi luoghi sotterranei e dalla storia della loro costituzione, Hugo associa una riflessione sull'igiene dell'aria e l'acqua, sull'economia e la pianificazione urbana come condizioni per il progresso sociale.

\section{LA DEPURAZIONE DELLE ACQUE}

${ }^{23}$ Arrivarono a Mara, ma non potevano bere le acque di Mara, perché erano amare. ${ }^{25}$ [Mosè] invocò il Signore, il quale gli indicò un legno. Lo gettò nell'acqua e l'acqua divenne dolce.

\section{Es 15, 23-25}

\subsection{Lo sviluppo pre-moderno della purificazione dell'acqua}

La tecnologia del collettamento dei reflui non è ovviamente sufficiente a garantire la sicurezza ambientale e sanitaria negli agglomerati urbani, poiché comunque tutto viene convogliato in acque superficiali che sono state usate da sempre come fonte di acque potabili, per l'igiene personale e per l'irrigazione. L'unica soluzione possibile è la depurazione delle acque reflue, cioè l'abbattimento delle sostanze chimiche o dei patogeni che, una volta diffusi nell'ambiente, possono presentare un rischio per gli ecosistemi e per gli essere umani.

Molti considerano l'episodio narrato al cap. 15 dell'Esodo (Fig. 3) il primo esempio di potabilizzazione delle acque e lo interpretano come un processo di scambio ionico in grado di sottrarre il magnesio, fonte principale del sapore amaro, mediante l'uso di specifici arbusti [8].

Nell'antichità molti autori (ad es. Plinio il Vecchio nella Naturalis historia) discussero metodi per dissalare l'acqua di mare. Traduttori arabi attribuirono ad Aristotele l'affermazione che l'acqua di mare diventa dolce per filtrazione attraverso le pareti di vasi di cera o di argilla, una storia ripetuta da molti commentatori fino al tardo Medioevo. 


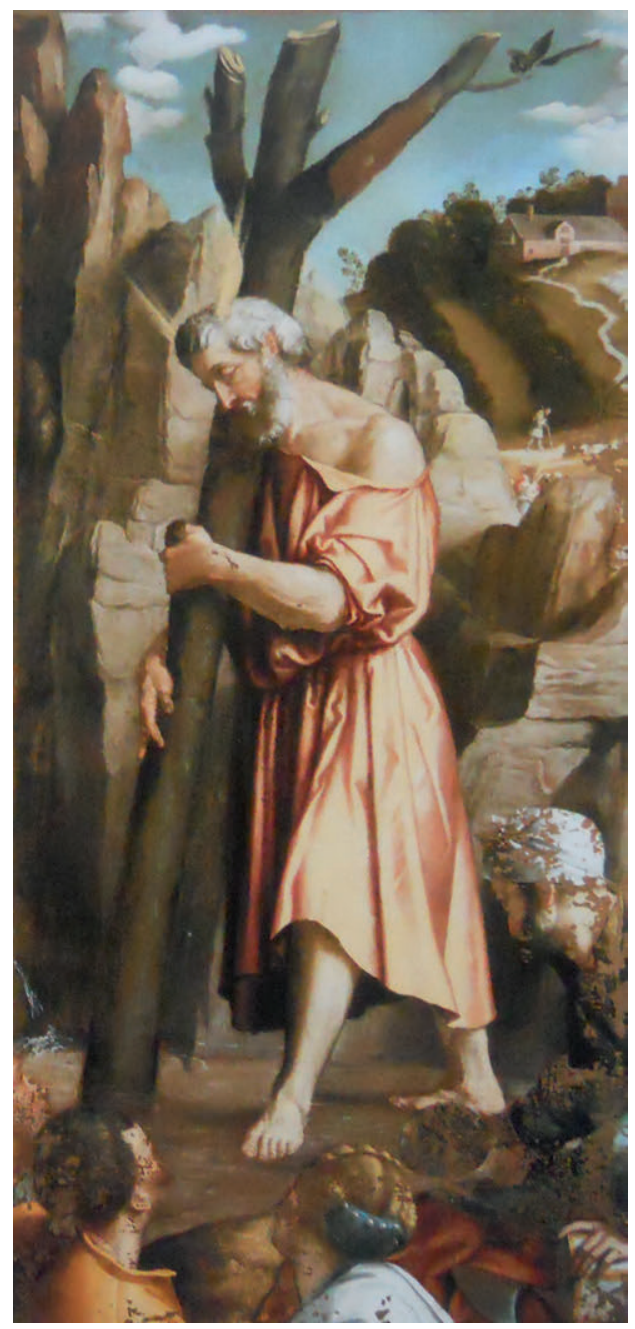

Fig. 3. Moretto da Brescia, Mosè addolcisce le acque di Mara.

Olio su tela, Collezione Privata, Brescia.

I Romani nei loro acquedotti sfruttavano ampiamente i processi di sedimentazione/decantazione nelle piscinae limariae che permettevano di evitare l'occlusione delle condutture e mantenevano salubre l'acqua fluente.

Tutta questa esperienza empirica trovò poi una sistematica appli- 
cazione nel trattamento di acque potabili e reflue a partire dal XVII sec. Nel 1685 il fisico italiano Antonio Porzio inventò un filtro che consisteva in un'unità di sedimentazione e una di filtraggio a sabbia. Nel 1746 vi fu il primo brevetto per un sistema di filtraggio delle acque, poi applicato alle abitazioni a partire dal 1750 .

Nel 1806 a Parigi fu messo in operazione un grande impianto per il trattamento dell'acqua. L'acqua sedimentava per 12 ore, prima di essere filtrata. I filtri erano costituiti da sabbia e carbone da legna e venivano sostituiti ogni sei ore. Nel 1827, l'inglese James Simpson costruì un filtro a sabbia per la depurazione dell'acqua potabile.

\subsection{La nascita della moderna tecnologia di depurazione delle acque}

La comprensione dei meccanismi di depurazione operanti in questi sistemi crebbe nel XIX sec. di pari passo con lo sviluppo della chimica analitica e soprattutto con l'esplosione degli studi di microbiologia. Era ormai chiaro che il solo collettamento dei reflui urbani per scaricarli in acque superficiali possibilmente a valle dell'abitato non evitava la diffusione epidemica dei patogeni, specie laddove la portata del corpo idrico recettore era paragonabile a quella degli scarichi immessi. Le fosse settiche perdenti, ampiamente usate fin dall'antichità e molto diffuse anche nel Medioevo specie nelle comunità monastiche e nei castelli, non erano una soluzione depurativa efficace in aree urbane intensamente abitate perché inquinavano i pozzi utilizzati per il prelievo di acqua potabile e dovevano essere svuotate molto frequentemente e smaltite sversandole nei fiumi o utilizzandole come ammendante nei campi.

Alla fine del XIX sec., a Berlino, risalgono le prime osservazioni sulla proprietà di auto-depurazione dei fiumi per ossidazione chimica spontanea di inquinanti organici e inorganici, ma ci vollero alcuni decenni perché si giungesse ad una visione condivisa del ruolo dei batteri nei meccanismi catabolici e anabolici coinvolti nei processi di degradazione [9]. I primi tentativi di risolvere il problema dei reflui urbani venne effettuato sempre a Berlino nell'ultimo decennio del secolo deviando gli scarichi verso "campi di spandimento", sfruttando la capacità di adsorbimento, filtrazione e degradazione microbica del suolo. Questa tecnica si diffuse in Germania a tal punto che sono attive da decenni alcune aree agricole (ad es. a Braunschweig) ora utilizzate per la ricarica artificiale della falda mediante infiltrazione di reflui urbani dopo opportuna depurazione. 
L'origine di questa tecnica di smaltimento e riuso dei reflui a scopo di fertilizzazione si può far risalire alle coltivazioni di marcite delle Abbazie a sud di Milano, ma il suo sviluppo portò nei primi anni del XX sec. alla costruzione di campi artificiali con contenimenti costruiti in muratura allo scopo di massimizzare la capacità filtrante dei suoli.

Parallelamente iniziarono gli studi per sistemi di trattamento dei reflui che lavorassero in batch o in flusso continuo e che sono tuttora alla base delle tecnologie adottate nei depuratori tradizionali.

Le prime applicazione si basarono su sistemi cosiddetti primari basati sulla rimozione di solidi più pesanti per sedimentazione di gravità, tra i quali il più celebre è il cono Imhoff disegnato nel 1906 e tuttora in uso [10]. Depuratori primari, cioè col solo sedimentatore, sono stati lo standard di depurazione anche nei paesi avanzati fino agli anni '70, quando sono stati lentamente sostituiti da sistemi cosiddetti secondari. Il trattamento secondario utilizza microrganismi per convertire la sostanza organica nelle acque reflue in anidride carbonica, acqua ed energia per la crescita della biomassa microbica. Esistono due tipi base di trattamento secondario: a biofilm adeso ad una superficie o a biomassa sospesa detto anche a fango attivo. I primi impianti a biofilm adeso su filtro vennero sperimentati alla fine del XIX sec. in Gran Bretagna e si diffusero in molte città del Regno Unito fin dagli anni venti. Nella stessa nazione vennero brevettati e sperimentati i primi impianti a flusso continuo con trattamento a fango attivo con insufflazione di aria a partire dagli anni '20. Questa tipologia di trattamento delle acque si diffuse lentamente nei paesi più sviluppati finché nel 1972 il Clear Water Act degli Stati Uniti lo impose come lo standard di trattamento delle acque. Da questo momento nasce la storia moderna del trattamento delle acque reflue [10].

4. PRESENTE E FUtURO DEL TRATTAMENTO DELLE ACQUE REFLUE: DA PROBLEMA A RISORSA NELL'OTTICA DELL'ECONOMIA CIRCOLARE

\subsection{I sistemi di trattamento biologici}

Dagli anni '70 si diffusero in tutto il mondo impianti di depurazione basati sulla degradazione biologica delle sostanze organiche e inorganiche di origine metabolica. Lo schema prevalente negli impianti delle grandi città è stato quello della sequenza di vasche di sedimenta- 
zione e impianti di trattamento biologico con biomassa sospesa e ricircolo del fango attivo. Uno dei problemi fondamentali affrontati da questi impianti è stato quello di abbattere la concentrazione in uscita di composti azotati e fosforati che davano luogo a significativi processi di eutrofizzazione nei corpi idrici recettori con ripercussioni significative anche nell'ambiente marino.

La rimozione del fosforo dall'acqua reflua è stata effettuata mediante l'incorporazione di fosfati in solidi sospesi e la successiva rimozione da tali solidi. Il fosforo può essere incorporato sia in solidi biologici, cioè nella biomassa microbica, che in precipitati chimici, mediante opportuni flocculanti.

L'azoto, presente nei reflui in prevalenza come ammoniaca o ammine, deve essere sottoposto ad un trattamento biologico ossidativo di nitrificazione (cioè la trasformazione da ammoniaca a nitrato), con l'ausilio di aria insufflata, e successiva denitrificazione in ambiente anaerobico per ridurre il nitrato ad azoto gassoso liberabile in atmosfera.

L'eutrofizzazione è stato il problema ambientale dominante nel campo della protezione delle acque fino almeno agli anni '90, quando esplose l'emergenza per la produzione elevata di mucillagini nell'alto Adriatico da parte di alghe fertilizzate dall'apporto di nutrienti del bacino padano tramite il fiume Po. Una delle cause principali di questa situazione non controllabile venne indicata nell'assenza di impianti di trattamento dei reflui urbani di Milano che entrarono in funzione solo nel 2005.

Per capire l'importanza della depurazione mostro un esempio di una ricerca condotta da un gruppo di ricerca del mio istituto (Istituto di Ricerca sulle Acque, IRSA-CNR), guidato dal dott. Tartari, per la misurazione del livello di fosforo nel lago di Pusiano nel corso degli ultimi 30 anni [11]. Come detto in precedenza il fosforo funge da fertilizzante per la crescita di biomassa algale che porta al fenomeno conosciuto come eutrofizzazione. Il problema grave è che la biomassa algale man mano che muore deposita al fondo e consuma ossigeno per la sua degradazione, portando il lago in breve tempo in condizioni anossiche, cioè privo di ossigeno e quindi di vita. Questa era la situazione ed il destino 30 anni fa di tutti i laghi prealpini lombardi non profondi. Nel caso del lago di Pusiano, studiato dall'IRSA, il collettamento circumlacuale dei reflui verso un nuovo impianto di trattamento a Merone ha portato ad una notevole riduzione del carico inquinante a lago (Fig. 4) [12]. Il problema è che ad un certo punto, benché sia cresciuta la percentuale di popolazione trattata il fosforo non diminuisce più: uno stu- 
dio recente del nostro istituto ha dimostrato che una delle sorgenti di questo carico residuo sono gli scolmatori di piena: quando piove, poiché il depuratore e le tubazioni non possono sopportare il carico idraulico, parte degli scarichi vengono direttamente scolmati, cioè scaricati nei torrenti che vanno al lago: Questo ci dice quanto sia urgente mettere in atto una soluzione non difficile dal punto di vista tecnico come la separazione delle acque di fognatura da quelle di dilavamento.

LAGO DI PUSIANO: EVOLUZIONE DEL FOSFORO 1984-2008

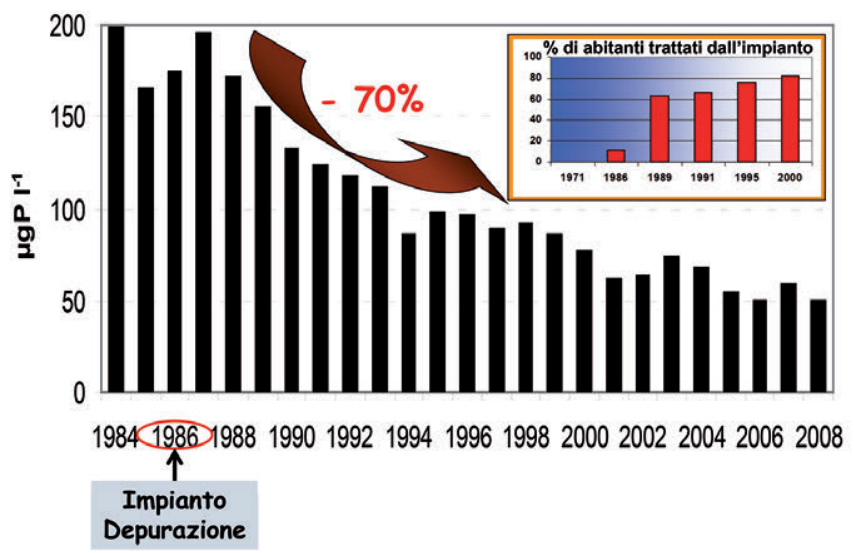

Fig. 4. Evoluzione temporale delle concentrazioni di fosforo (in $\mu \mathrm{g} P / \mathrm{L}$ ) nel Lago di Pusiano, Lombardia.

\subsection{I sistemi di trattamento avanzati nell'economia circolare dell'acqua}

L'aumento delle conoscenze sul destino delle molecole provenienti da attività umane e immesse nell'ambiente, grazie anche al grande sviluppo tecnologico delle strumentazioni analitiche, ha evidenziato il rischio per l'uomo e gli ecosistemi di un grande numero di sostanze, anche di uso domestico e quotidiano, detti convenzionalmente "inquinanti emergenti" che sono continuamente scaricati nei fiumi europei o raggiungono le falde utilizzate per il prelievo di acqua destinata al consumo umano. Oggi il problema principale della depurazione è abbatt ere il carico inquinante di una città o di una vasta area cercando di riversare nel corpo idrico recettore un'acqua che non comporti rischi per l'ecosistema acquatico. Gli attuali depuratori standard sono basati sul principio della sedimentazione e degradazione biologica della materia organica e in parte dei metalli, men- 
tre sono poco efficaci con le molecole di sintesi scaricate dall'uso comune come farmaci o prodotti per la cura della persona. Lo sviluppo attuale è di aggiungere stadi di trattamento ulteriori (cosiddetti terziari) mirati a queste sostanze, in modo da produrre acqua di qualità "potabile". I trattamenti cosiddetti terziari si basano su tecnologie di ossidazione avanzata e adsorbimento/filtrazione, spesso proposti in una sequenza di trattamenti combinata disegnata su misura sulla tipologia e la composizione di acque da trattare e soprattutto in funzione dell'uso dell'acqua trattata. Le tecniche di ossidazione avanzata si basano su una combinazione di agenti ossidanti (ad es. $\mathrm{O}_{3}$ e $\mathrm{H}_{2} \mathrm{O}_{2}$ ) talvolta accoppiati con altre specie chimiche (catalizzatori metallici, es. $\mathrm{Fe}^{2+}$ ) o con agenti fisici (radiazione UV o ultrasuoni) in modo da incrementare l'efficienza di eliminazione dei contaminanti più recalcitranti per mezzo di un aumento della formazione di radicali liberi. L'obiettivo è quello di degradare le molecole originarie a composti più semplici o più eliminabili per mezzo di un successivo trattamento di adsorbimento su una fase solida attiva, come il carbone o di filtrazione/adsorbimento come l'osmosiinversa o ultrafiltrazione: questi sistemi possono essere accoppiati ad un trattamento biologico secondario effettuato però con una biomassa molto più concentrata adesa sulla membrana.

Nell'ottica dell'economia circolare, fortemente incentivata dall'Unione Europea, l'obiettivo di questi trattamenti è quello di garantire in uscita ai depuratori acqua trattata utilizzabile per altre attività umane, come l'irrigazione agricola e urbana e la ricarica di falda mediante infiltrazione sul suolo, nell'ottica di garantire un quantitativo costante di acqua di qualità sufficiente a contrastare la crescente richiesta di acqua a fronte di una sempre più ridotta disponibilità, soprattutto nelle regioni mediterranee, a causa dei cambiamenti climatici in corso.

Il riutilizzo delle acque reflue urbane - sia non trattate sia trattate - è stato praticato per molti secoli con l'obiettivo di deviare le deiezioni umane al di fuori degli insediamenti urbani. Il riutilizzo dell'acqua come attività pianificata è iniziata circa un secolo fa con l'uso di effluenti trattati per l'irrigazione dei parchi pubblici. Da allora le applicazioni di riutilizzo dell'acqua non potabile sono cresciute in modo sostanziale dall'irrigazione del paesaggio urbano all'irrigazione di colture alimentari, dall'uso come acqua di raffreddamento, negli impianti di autolavaggio, antincendio, fontane pubbliche, come mezzo di incremento delle portate fluviali alle barriere di intrusione dell'acqua di mare. Con l'introduzione dei trattamenti terziari e il conseguente miglioramento della qualità degli effluenti e la concomitante diminuzione della disponibilità 
locale di acqua dolce, il riutilizzo delle acque reflue urbane trattate è stato preso in considerazione anche per aumentare le forniture di acqua potabile fin dagli anni ' 60 con applicazioni pionieristiche negli Stati Uniti d'America (USA) e in Namibia. Oggi, il riutilizzo di acqua potabile pianificato è riconosciuto in tutto il mondo come una componente sempre più importante della gestione delle risorse idriche regionali con un numero crescente di progetti consolidati. I progetti di riutilizzo dell'acqua potabile pianificati sono caratterizzati come diretti o indiretti [13]. Città come Singapore o Windhoek (Namibia) immettono in modo diretto negli acquedotti una aliquota significativa di acqua in uscita dai depuratori urbani dopo una sequenza di trattamenti avanzati. Il riutilizzo indiretto di acqua potabile viene definito invece come l'aggiunta intenzionale di acque reflue trattate ad un sistema di accumulo naturale o artificiale utilizzato per la fornitura di acqua potabile. Queste aree di accumulo possono essere una falda acquifera naturale o un serbatoio lacuale artificiale con l'intento di fornire ritenzione, attenuazione aggiuntiva dei contaminanti e miscelazione prima dell'uso come acqua potabile. Città come Las Vegas, in pieno deserto, ricaricano già i bacini di approvvigionamento idrico con l'acqua trattata dai depuratori urbani.

Il nostro istituto IRSA-CNR ha recentemente partecipato al progetto europeo FRAME per lo sviluppo di tecnologie e protocolli di riutilizzo potabile attraverso la ricarica delle acque sotterranee mediante infiltrazione di acqua trattata attraverso la zona vadosa per dispersione superficiale.

\section{CONCLUSIONI}

Il campo di ricerca degli studi ambientali sull'acqua ed in particolare sulle tecnologie di depurazione mostra chiaramente come oggi sia del tutto privo di senso cercare una linea di demarcazione o una contrapposizione tra ricerca pura e applicata, che invece sono intrecciate e al massimo complementari, come la storia del nostro istituto ha sempre messo in pratica dalla sua fondazione. Colgo l'occasione perciò per chiudere con le parole di Leonardo, grande osservatore del mondo naturale, ma anche ricercatore di soluzioni a problemi pratici:

Quando tu metti insieme la Scienza de' moti dell'acqua, ricordati di mettere, di sotto a ciascuna proposizione, li sua giovamenti, a ciò che tale scienza non sia inutile [14] 


\section{RIFERIMENTI BIBLIOGRAFICI}

1. La Sacra Bibbia, Conferenza Episcopale Italiana, Roma, 2008.

2. Bandini F, E fu sera e fu mattina: primo giorno. I miti della creazione e delle origini della vita tra eros e ethos, Alinea Editrice, Firenze, 2006.

3. Alighieri D, Tutte le Opere, Sansoni Editore, Firenze, 1965.

4. Pace P, Acquedotti di Roma e il De aquaeductu di Frontino, Consiglio Nazionale delle Ricerche, Roma, 2010.

5. Mitchell PD, Human parasites in the Roman World: health consequences of conquering an empire, Parasitology, 2017: 144: 48-58.

6. Boccaccio G, Decameron, Le Monnier ed., Firenze, 1951-1952.

7. Hugo V, I Miserabili, Garzanti ed., Milano, 1981.

8. Ettre LS, Was Moses the First Chromatographer? Chromatography in the Ancient World, LCGC North America, 2006: 24: 1280-1283.

9. Wiesmann U, Choi IS, Dombrowski EM, Fundamentals of Biological Wastewater Treatment. Wiley-VCH Verlag GmbH \& Co., Weinheim, Germany, 2007.

10. Lofrano G, Brown J, Wastewater management through the ages: A history of mankind, Science of the Total Environment 2010: 408: 5254-5264.

11. AAVV, Qualità delle acque lacustri in Lombardia - $1^{\circ}$ Rapporto Osservatorio dei Laghi Lombardi 2004, Regione Lombardia, ARPA Lombardia, Fondazione Lombardia per l'Ambiente e IRSA/CNR, Milano, 2005

12. Sangalli L, Evoluzione della qualità chimica e fisica nei laghi di Alserio e Pusiano, Parco Regionale della Valle del Lambro, www.parcovallelambro.it

13. Drewes JE, Horstmeyer N. Recent Developments in Potable Reuse, Springer International Publishing, Switzerland, 2015.

14. Leonardo da Vinci, Aforismi, novelle e profezie, Newton Compton ed., Roma, 1993. 